\title{
Characterization of Cl-Implanted Al Films with STEM-EDS Spectral Imaging
}

\author{
D. Elswick*, J. Hren*, P. Kotula**, N. Missert** \\ * North Carolina State University \\ Raleigh, NC 27695-7907 \\ ** Sandia National Laboratories \\ Albuquerque, NM 87185-1415
}

The initiation stages of corrosion in aluminum have been studied extensively using electrochemical techniques. Several models focus on the morphology and structure of defects in the metal or at the metal-oxide interface. Another important consideration is the role of chloride. The primary goal of this research is to utilize several advanced characterization techniques to investigate the role of $\mathrm{Cl}^{-}$in pit initiation. In particular, atomic force microscopy (AFM), Automated X-ray Spectral Image Analysis (AXSIA), Z-contrast imaging, and electron energy loss spectroscopy (EELS) were used to explore film morphology, $\mathrm{Cl}$ spatial distribution and changes in chemical bonding as a function of $\mathrm{Cl}$ concentration. Previous studies utilized various sample geometries and polarization in $\mathrm{NaCl}$ solutions to incorporate $\mathrm{Cl}$, while the current study employs ion implantation as the means to introduce chlorine. Polycrystalline 99.9999\% Al thin films were deposited using electron beam evaporation followed by exposure to ultra-high purity oxygen and then ambient atmosphere to form a uniform $5 \mathrm{~nm}$ oxide. These films were then implanted using $35 \mathrm{keV} \mathrm{Cl}^{+}$ions with fluences of 1, 3, 5 and $7 \mathrm{e} 16 \mathrm{Cl} / \mathrm{cm}^{2}$. Upon anodic polarization in sulfate solution, pitting occurred at lower potentials as $\mathrm{Cl}$ concentration increased, indicating a higher susceptibility to pitting with higher $\mathrm{Cl}$ concentrations. To gain an insight into the role of $\mathrm{Cl}$ and pitting phenomena, AFM images of surface morphology and both plan-view and cross-sectional TEM samples were made of the implanted thin films.

The AFM images in Figure 1 show the change in surface morphology as a function of ion implantation fluence or $[\mathrm{Cl}]$. As the fluence is increased, the boundaries become more pronounced and defined with a change in topography. These features are likely a result of increasing surface sputtering during the implantation process and may also contribute to pitting susceptibility. The STEM dark field image in Figure 2 is a cross-section of the $5 \mathrm{e} 16 \mathrm{Cl} / \mathrm{cm}^{2}$ sample showing damage from implantation. Spectral imaging utilizes a fine probe to map out the EDS spectra and allows a reconstruction of a chemical map (or phases) with excellent spatial resolution (1-2 nm). AXSIA was performed in the red box region of the DF image. The component spectrum is also given, identifying four unique components. These four components are shown in a reconstructed RGB overlay with blue, green, red and magenta corresponding to $\mathrm{SiO}_{2}$ substrate, $\mathrm{Al}$ thin film, Cl-rich region and the oxide respectively. The chlorine-rich phase is distributed across the lateral surface with a fairly consistent implant depth of $\sim 75 \mathrm{~nm}$. When compared to the $7 \mathrm{e} 16 \mathrm{Cl} / \mathrm{cm}^{2}$ implanted specimen, a similar spectrum image is obtained but with the Cl-rich component appearing more clustered.

AXSIA on the cross-sections helped determine the $\mathrm{Cl}$ depth profile with some uncertainty in its planar distribution. This technique was then used on the plan-view specimens with results shown in Figure 3. The spectral image area in Figure 3 is 200 by $200 \mathrm{~nm}$ for both samples. This analysis demonstrates the random planar distribution of $\mathrm{Cl}$ for both implanted specimens and shows an increasing cluster size with higher fluence. Z-contrast images were also obtained where diffuse 
bright regions outlined the boundaries. This effect corresponded to the domains seen in AFM and may correlate to a local thickness increase. EELS was performed within the boundaries of the two contrasting regions and found that this effect was not related to a local increase in $\mathrm{Cl}$ concentration.

AXSIA and Z-contrast imaging have proven to be very powerful techniques to help understand the role of $\mathrm{Cl}$ in corrosive pitting. It is evident that within the metal, $\mathrm{Cl}$ is uniformly distributed at low concentrations and does not segregate to grain boundaries for any of the concentrations investigated. The use of electron loss near edge spectroscopy (ELNES) will also be used to look at the O k-edge fine structure to help determine the chemical influence of the $\mathrm{Cl}$ within the oxide. The combination of electrochemical data and sophisticated microanalytical techniques is expected to yield a more complete understanding of the role of $\mathrm{Cl}$ on the initiation stages of pitting in aluminum.

This work is supported by US DOE Division of Materials Science and Engineering, Office of Basic Energy Sciences, and by Sandia National Laboratories. Sandia is a multiprogram laboratory by Sandia Corporation, a Lockheed Martin Company, for the US Dept. of Energy's National Nuclear Security Administration under contract DE-AC0494AL85000.
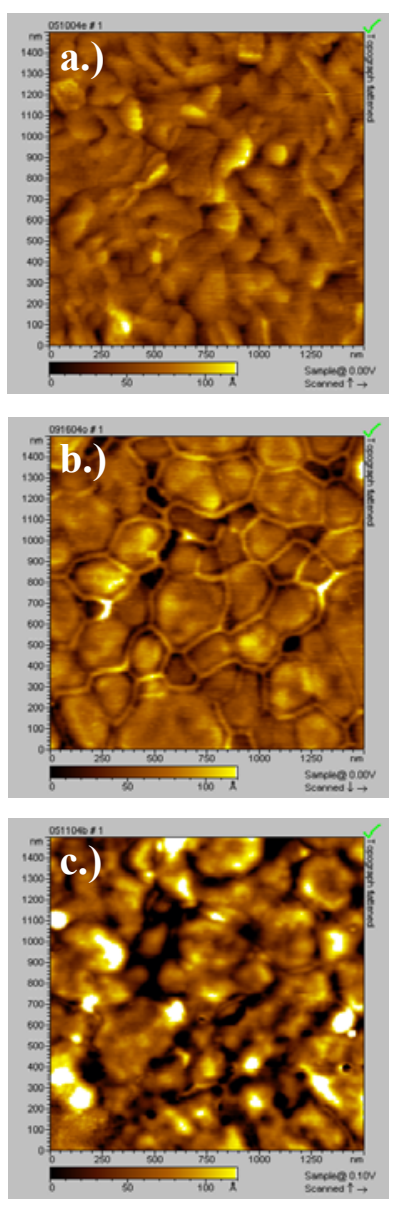

Figure 1. AFM of Climplanted $\mathrm{Al}$ thin films. a.) No implant b.) $5 \mathrm{e} 16 \mathrm{Cl} / \mathrm{cm}^{2}$ and c.) $7 \mathrm{e} 16 \mathrm{Cl} / \mathrm{cm}^{2}$

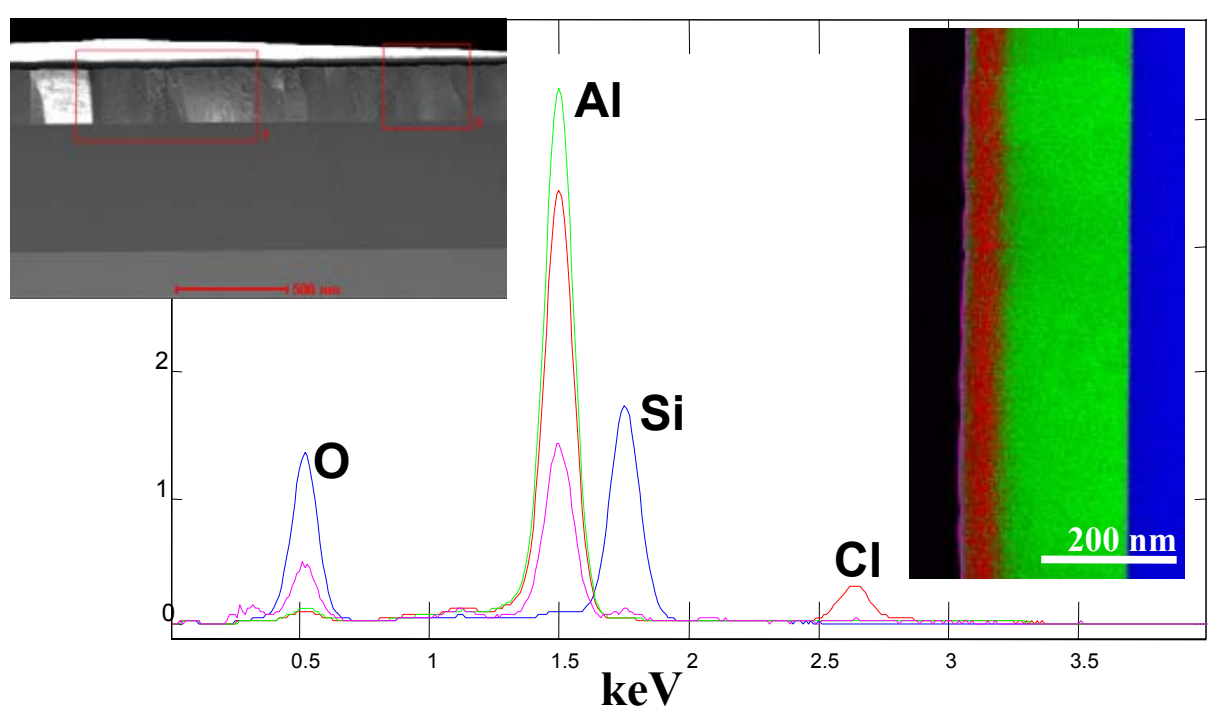

Figure 2. Results from a FIB cross-section of an Al thin film implanted with $5 \mathrm{e} 16 \mathrm{Cl} / \mathrm{cm}^{2}$. Inset shows a STEM DF with the area selected for AXSIA. Component spectra from analysis and corresponding RGB component overlay are also shown.
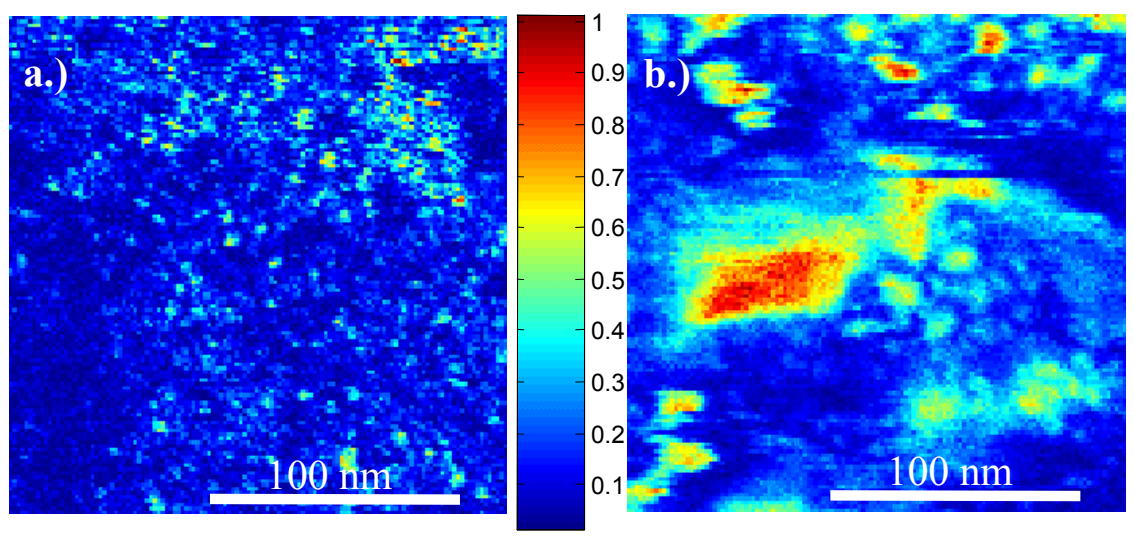

Figure 3. AXSIA $\mathrm{Cl}$ component images from plan-view $\mathrm{Al}$ thin film samples implanted with $35 \mathrm{keV} \mathrm{Cl}^{+}$a.) $5 \mathrm{e} 16 \mathrm{Cl} / \mathrm{cm}^{2}$ b.) $7 \mathrm{e} 16 \mathrm{Cl} / \mathrm{cm}^{2}$ 